United Kingdom; ${ }^{2}$ Cambridge University, Clinical Neurosciences, Cambridge, United Kingdom; ${ }^{3}$ King's College Hospital, Rheumatology, London, United Kingdom

Background: Axial spondyloarthritis (axSpA) is a chronic inflammatory disease predominantly involving the axial skeleton and sacroiliac joints. Although the exact aetiology remains largely unknown, there is thought to be an immunedriven element. Vitamin $D$ deficiency has been associated with a number of autoimmune diseases and is thought to play an important role in modulating the immune system. Low vitamin D levels may contribute to the development and progression of $\operatorname{axSpA}{ }^{1}$

Objectives: To study the possible associations between low vitamin D and disease activity in axSpA.

Methods: A systematic literature search using Medline, Embase and Cochrane was performed using MESH search terms "ankylosing spondylitis", "axial spondyloarthropathy" and "vitamin D". Articles examining disease activity measured by BASDAI, ASDAS-CRP, ESR and CRP identified through title/abstract screening, were included in the study, with relevant information extracted.

Results: Out of 495 articles identified from the initial search, 19 observational studies which were mostly $(89 \%)$ cross-sectional studies were identified. There was considerable heterogeneity between studies, including in the definition of vitamin D deficiency, latitude where study took place and seasonal variation. Vitamin D levels were often lower in patients with axSpA compared to controls. Seventeen studies found no association with vitamin $\mathrm{D}$ deficiency and disease activity. The exceptions included one study which measured serum vitamin D receptor levels as opposed to serum $25(\mathrm{OH})$ D or 1,25 $(\mathrm{OH}) 2 \mathrm{D}$ concentrations, and another study whose recruitment occurred over four years and therefore seasonal variation may conflict results. Patients taking NSAIDs or anti-TNF had no difference in vitamin D levels.

Conclusion: Vitamin D deficiency is more prevalent in axSpA but does not seem to associate with increased disease activity. Longitudinal studies are required to better define these links.

References:

[1] Sizheng Z, et al. Systematic review of association between vitamin D levels and susceptibility and disease activity of ankylosing spondylitis. Rheumatology 2014; 53:1595-1603.

Disclosure of Interests: None declared

DOI: 10.1136/annrheumdis-2020-eular.4684

\section{AB0674 CLINICAL AND IMAGING FEATURES IN SPONDYLOARTHRITIS PATIENTS WITH AND WITHOUT HLA-B27 AND HLA-B51}

F. Carubbi ${ }^{1}$, A. Alunno ${ }^{2}$, A. Conforti ${ }^{1}$, A. F. Bonifacio ${ }^{2}$, P. Cipriani ${ }^{1}$, R. Gerli $^{2}$, R. Giacomelli ${ }^{1} .{ }^{1}$ Rheumatology Unit, L'Aquila, Italy; ${ }^{2}$ Rheumatology Unit, Perugia, Italy

Background: Despite being commonly expressed in the general population, the human leucocyte antigen (HLA)-B27 allele strongly increases the susceptibility to develop spondyloarthritis (SpA). Likewise, the association between the HLAB51 allele and the development of Behçet's disease is well documented. However, the exact mechanisms responsible for their pathologic role are still a matter of debate. Moreover, anecdotal reports show an association between HLA-B51 and the clinical spectrum of SpA.

Objectives: To investigate the clinical and imaging findings of SpA patients according to the absence or presence of HLA-B27 or HLA-B51.

Methods: We retrospectively analyzed 236 patients with axial or peripheral SpA, according to the ASAS criteria, referring to two tertiary Rheumatology Clinics between 2017 and 2019. All patients had been tested for HLA-B alleles. Patients with HLA-B51 haplotype and fulfilling the criteria for Behçet's disease were excluded.

Results: Table 1 shows demographic and clinical features of patients, according with the HLA-B haplotype (neither HLA-B27 nor -B51, double negative; positive for HLA-B27 only, positive for HLA-B51 only). Inflammatory low back pain and sacroiliitis, assessed by X-ray or magnetic resonance imaging (MRI), were more prevalent in double negative and HLA-B27 patients, compared to HLA-B51 patients. In this regard, the presence of HLA-B51 was negatively associated with axial manifestations at onset (OR $0.347,95 \% \mathrm{Cl} 0.200-0.604, \mathrm{p}<0.0001$ ) and in the course of the disease (low back pain: OR $0.395,95 \% \mathrm{Cl} 0.225-0.689 \mathrm{p}<0.0001$; sacroiliitis on imaging: OR $0.342 \mathrm{Cl} 0.189-0.619 \mathrm{p}<0.0005)$. When considering extra-articular manifestation, aphtous lesions were more prevalent in patients with HLAB51 $(p<0.0001)$, inflammatory bowel diseases in the double negative group $(p=0.0006)$, and increased $C$-reactive protein in double negative and HLAB27 patients $(p=0.02)$

Table 1.

\begin{tabular}{|c|c|c|c|c|c|}
\hline & $\begin{array}{c}\text { All } \\
(236 \\
\text { patients })(\end{array}$ & $\begin{array}{c}\text { Double- } \\
\text { negative } \\
\text { (101 patients) }\end{array}$ & $\begin{array}{l}\text { HLA-B27 } \\
\text { (53 patients) }\end{array}$ & $\begin{array}{c}\text { HLA-B51 } \\
\text { (82 patients) }\end{array}$ & $p$ value \\
\hline Female (\%) & $167(71)$ & $73(72)$ & $28(53)$ & $66(60)$ & 0.0024 (cumulative) \\
\hline $\begin{array}{l}\text { Age, years } \\
\quad(\text { mean } \pm S D)\end{array}$ & $53.7 \pm 13.3$ & $56.4 \pm 12.7^{\#}$ & $48.2 \pm 14.4^{\$}$ & $54 \pm 12.7$ & $\begin{array}{l}\#<0.01 \text { vs HLA-B27 } \\
\$<0.05 \text { vs HLA-B51 }\end{array}$ \\
\hline $\begin{array}{l}\text { Age at diagnosis, } \\
\quad \text { years }(\operatorname{mean} \pm S D)\end{array}$ & $48 \pm 14.5$ & $50.3 \pm 13^{*}$ & $40.8 \pm 15.5^{\S}$ & $50.2 \pm 14.3$ & $\begin{array}{l}{ }^{*}<0.001 \text { vs HLA-B27 } \\
\S<0.001 \text { vs HLA-B51 }\end{array}$ \\
\hline $\begin{array}{l}\text { Time from symptoms } \\
\text { to diagnosis, years } \\
\text { (mean } \pm \text { SD) }\end{array}$ & $3.8 \pm 5.7$ & $3.6 \pm 4.8$ & $3.2 \pm 3.7$ & $4.4 \pm 7.5$ & ns \\
\hline Type of onset: & & & & & 0.0031 (cumulative) \\
\hline -Axial (\%) & $129(55)$ & $67(66)$ & $31(59)$ & $31(38)$ & \\
\hline -Peripheral (\%) & $63(27)$ & $19(19)$ & $12(23)$ & $32(39)$ & \\
\hline $\begin{array}{l}\text {-Axial and } \\
\quad \text { peripheral (\%) }\end{array}$ & $44(19)$ & $15(15)$ & $10(19)$ & $19(23)$ & \\
\hline
\end{tabular}

Conclusion: The presence of HLA-B51 identifies a subgroup of SpA patients with peculiar features compared to double-negative or HLA-B27 SpA patients HLA-B51-related SpA may be an additional condition to be included in the SpA spectrum.

References:

[1] Bodis G, et al. Role of Human Leukocyte Antigens (HLA) in Autoimmune Diseases. Rheumatol Ther 2018;5:5-20.

[2] Rudwaleit M, et al. The development of Assessment of SpondyloArthritis international Society classification criteria for axial spondyloarthritis (part II): validation and final selection. Ann Rheum Dis 2009;68:777-83.

[3] Rudwaleit M, et al. The Assessment of SpondyloArthritis International Society classification criteria for peripheral spondyloarthritis and for spondyloarthritis in general. Ann Rheum Dis 2011;70:25-31.

[4] Maksymowych WP, et al. MRI lesions in the sacroiliac joints of patients with spondyloarthritis: an update of definitions and validation by the ASAS MRI working group. Ann Rheum Dis. 2019;78:1550-1558.

Disclosure of Interests: Francesco Carubbi Speakers bureau: Francesco Carubbi received speaker honoraria from Abbvie and Celgene outside this work. Alessia Alunno: None declared, Alessandro Conforti: None declared, Angelo Francesco Bonifacio: None declared, Paola Cipriani Grant/research support from: Actelion, Pfizer, Speakers bureau: Actelion, Pfizer, Roberto Gerli: None declared, Roberto Giacomelli Grant/research support from: Actelion, Pfizer, Speakers bureau: Abbvie, Roche, Actelion, BMS, MSD, Ely Lilly, SOBI, Pfizer DOI: 10.1136/annrheumdis-2020-eular.6556

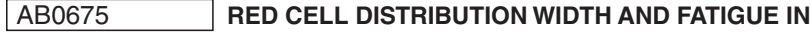 AXIAL SPONDYLOARTHRITIS: A SLEEPER SIGNAL}

S. Chatterjee ${ }^{1}$, C. Wincup ${ }^{2}$, A. Rahman ${ }^{2}$, R. Sengupta ${ }^{1} .{ }^{1}$ Royal National Hospital for Rheumatic Diseases, Bath, United Kingdom; ${ }^{2}$ Department of Rheumatology, University College London, London, United Kingdom

Background: Fatigue is a ubiquitous feature of autoimmune conditions, and axial Spondyloarthritis (axSpA) is no exception, with over $50 \%$ of patients reporting some degree of fatigue ${ }^{1}$. Erythrocyte size variability (as measured by red cell distribution width (RDW)) has been found to correlate with fatigue in a cohort of systemic lupus erythematous (SLE) patients and may reflect early iron deficiency $^{2}$. We investigate whether this finding holds true in ax $\mathrm{SpA}$.

Objectives: To investigate the relationships between fatigue, disease activity, and RDW (as a proxy for functional iron deficiency) in patients with axSpA.

Methods: Cross-sectional analysis performed on patients with axSpA, as defined by the Assessment of SpondyloArthritis international Society (ASAS) criteria, enrolled in a longitudinal data collection study from October 2017 until January 2020 in a single outpatient setting. Patients required a minimum of 1 set of patient-reported outcome measures (PROMs), including the completion of a Functional Assessment of Chronic Fatigue Illness Therapy (FACIT) Fatigue Scale v4 (numerical score between 0-52 with a lower score indicating greater fatigue), and Bath Ankylosing Spondylitis Disease Activity Index (BASDAI). C-reactive protein (CRP) as a biochemical marker of disease activity, as well as Haemoglobin $(\mathrm{Hb})$ and RDW performed within 3 months either side of a PROMs set were extracted and matched. Only one pair of matched data was selected per patient according to the least timeframe between a se of PROMs and bloods of interest (if multiple were available). Anaemia was defined according to World Health Organisation criteria, i.e., $\mathrm{Hb}<120 \mathrm{~g} / \mathrm{L}$ in 
females and $\mathrm{Hb}<130 \mathrm{~g} / \mathrm{L}$ in males. Non-parametric analysis of variables was performed using Spearman's rank correlation with significance defined at a p-value $<0.05$

Results: 63 patients were included in the analysis $(63.5 \%$ (40) male, mean time to diagnosis $11.46( \pm 9.04)$ years, $79.4 \%$ (50) HLA-B27 positive, $46 \%(29)$ current or ex-smokers). Blood parameters showed mean $\mathrm{Hb}$ of $139.6( \pm 16.03) \mathrm{g} / \mathrm{L}$, mean RDW of $13.55( \pm 1.46) \%$, mean CRP of $5.23( \pm 10.82) \mathrm{mg} / \mathrm{L}$. Mean BASDAI score of cohort was $3.69( \pm 2.02)$ and FACIT score $34.18( \pm 11.30)$. Mean absolute interval time difference between a PROMs set and bloods of interest was $16.14( \pm 41.11)$ days.

Univariate analysis showed a statistically significant, negative correlation between fatigue (FACIT) and disease activity (BASDAI), $(p<0.001 ; r=-0.63)$, but failed to demonstrate an association between fatigue and $\mathrm{Hb}, \mathrm{RDW}$, or CRP. Subgroup analysis of 51 patients, following exclusion of patients with anaemia (12), engendered a significant and moderately negative correlation between fatigue and RDW ( $p=0.02, r=-0.32$ ) (Figure 1), maintained a significant correlation between fatigue and BASDAI $(p<0.0001, r=-0.56)$ and showed a non-significant association between RDW and BASDAI $(p=0.07, r=0.25)$.

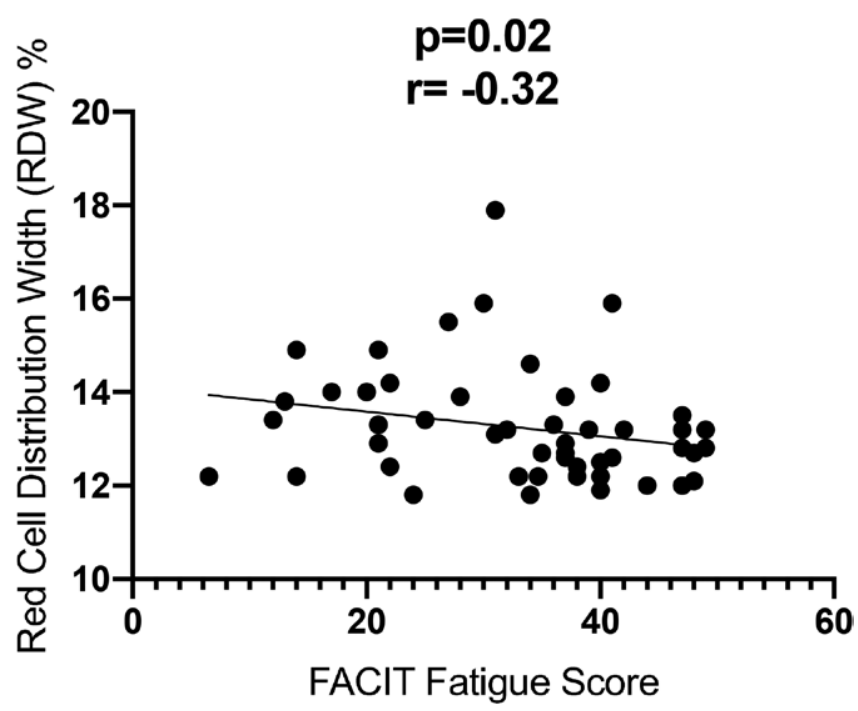

Figure 1. Negative correlation between FACIT score and RDW

Conclusion: These findings suggest that RDW may potentially represent a surrogate marker of disease activity in patients with axSpA. RDW may also be implicated in the multi-faceted aetiology of fatigue in axSpA patients, and may reflect functional iron deficiency. A recent cohort study of axSpA patients found anti-TNF therapy ineffective at alleviating fatigue in nearly $80 \%$ of patients ${ }^{1}$. Management of potential functional iron deficiency as reflected by RDW may offer an alternative treatment target for fatigue in these patients.

References:

[1] Bedaiwi M, Sari I, Thavaneswaran A, et al. J Rheumatol. 2015;42(12):2354.

[2] Wincup C, Parnell S, Cleanthous B, et al. Clin Exp Rheumatol. 2019 Sep-Oct;37(5):852-54.

Disclosure of Interests: Saion Chatterjee: None declared, Chris Wincup: None declared, Anisur Rahman: None declared, Raj Sengupta Grant/research support from: Research grants from UCB, Pfizer, Abbvie and Novartis, Speakers bureau: Received honoraria for giving talks from Abbvie, Biogen, UCB, Novartis, Pfizer DOI: 10.1136/annrheumdis-2020-eular.6588

\begin{tabular}{|l|l}
\hline AB0676 & THE ROLE OF ACPA AND ANA IN \\
SPONDYLOARTHRITIS: HOW THE AUTOIMMUNE \\
DYSREGULATION CAN AFFECT THE COURSE \\
OF DISEASE AND THERAPEUTIC SUCCESS OF \\
MONOTHERAPY DMARDS AND BDMARDS
\end{tabular}

D. Cici ${ }^{1}$, C. Rotondo ${ }^{1}$, A. Corrado ${ }^{1}$, S. Berardi ${ }^{1}$, N. Mansueto ${ }^{1}$, L. Lops ${ }^{1}$, F P. Cantatore ${ }^{1}{ }^{1}$ University of Foggia, Department of Medical and Surgical Science, Foggia, Italy

Background: Occasional findings of anti-citrullinated-protein-antibodies (ACPA) and anti-nuclear-antibodies (ANA) were rarely described in literature on Psoriatic Arthritis (PsA) and on Spondyloarthritis (SpA) in general. How these autoimmune dysregulations can affect the course of them is not yet understood.

Objectives: The aim of our study is to evaluate if the presence of ACPA and ANA can determine different disease subsets and influence the DMARDs monotherapy (methotrexate) drug survival (DSM) and b-DMARDs multi-failure patients (MF).
Methods: We conducted a retrospective study on patients with Psoriatic Arthritis (PSA) and Spondyloarthritis that fulfilled the ASAS and CASPAR criteria Patients with diagnosis of connective tissue disease and rheumatoid arthritis and patients $\leq 18$ years old were excluded from the study. For each patient, the following variables were considered: age, ACPA, ANA, time between arthritis onset and start of DMARDs (start-time), DSM, switch to b-DMARDs (sw-bDMARDs), arthritis subset (oligoarticular (OA), polyarticular (PA), enthesitis (EA) axial involvement $(\mathrm{Al})$ ), number of comorbidities (NC), Charlson Comorbidity Index (CCI).

Results: 150 patients (55\% with PsA and 45\% with another SpA) were included in the study. No differences were found in age, ANA rate, ACPA rate, start-time $\mathrm{OA}, \mathrm{PA}, \mathrm{EA}, \mathrm{Al}, \mathrm{NC}$ and $\mathrm{CCl}$ between the PsA and SpA groups.

In the whole group of patients, the ACPA+ subjects(11\%) had a significant increase of $\mathrm{NC}(2.47 \pm 1.5$ vs $1.6 \pm 1.4, \mathrm{p}=0.035)$, a trend to higher $\mathrm{CCl}$, to switch to b-DMARDs, and to be MF compared to those without ACPA. In the same group, the ANA+ patients (12\%) showed shorter DSM (233.5 wk \pm 45.9 vs $548.0 \mathrm{wk} \pm 56.8, \mathrm{p}=0.362$ ) with similar trend in each subgroup (PsA and $\mathrm{SpA}$ ).

In SpA group, the ACPA+ patients(6,3\%) had a trend to shorter DSM (269.0 weeks $\pm 125 \mathrm{vs} 603.96 \mathrm{wk} \pm 92.8, \mathrm{p}=0.492)$, to higher sw-bDMARDs, and to be $\mathrm{MF}$, higher $\mathrm{NC}$ and $\mathrm{CCl}$ compared to those without ACPA. No differences in clinical subset (OA, PA, EA, Al) were observed. In the same group the ANA+ patients had significant higher rate of PA $(100 \%$ vs $65 \%, p=0.026)$ rather than OA $(0 \%$ vs $35 \%, p=0.025)$. No significant differences were found in $\mathrm{NC}, \mathrm{CCl}, \mathrm{MF}$.

In the PsA group, ACPA+ patients showed a trend to develop PA and EA subsets, shorter DSM $(187.5 w \mathrm{wk} \pm 48.7$ vs $299.6 \mathrm{wk} \pm 31.4, \mathrm{p}=0.415)$, higher rate to sw-bDMARDs and to be MF. The ANA+ PsA patients had higher trend to develop $\mathrm{PA}$ and $\mathrm{Al}$ subsets rather than OA and EA. All ANA+ patients were MF ( $100 \%$ vs $42 \%, \mathrm{p}=0.046$ ).

Conclusion: The ACPA and ANA positivity in PsA and SpA patients could be suggestive of more severe clinical disease manifestation, higher frequency of comorbidities and lower predicted 10-year survival (CCI). Moreover, this autoimmune dysregulation could be associated with worse drug survival in monotherapy with methotrexate and higher chance to be MF. Therefore, they can be taken into account for clinical management of these patients.

Disclosure of Interests: None declared

DOI: 10.1136/annrheumdis-2020-eular.4526

\section{$\mathrm{AB} 0677$ \\ REVISING THE DEFINITION OF REACTIVE ARTHRITIS AND DIFFERENTIATION FROM UNDIFFERENTIATED SPONDYLOARTHRITIS}

F. Cosan ${ }^{1}$, O. M. Gedar ${ }^{2}{ }^{1}$ Bahcesehir University, Faculty of Medicine, Internal Medicine, Rheumatology, Istanbul, Turkey; ${ }^{2}$ Bahçeşehir University, Faculty of Medicine, Ophtalmology, Istanbul, Turkey

Background: Reactive arthritis (ReA) is defined by 1999 ACR criteria as arthritis preceding a bacterial genitourinary (GUS) or gastrointestinal (GIS) infection in 3 days -6 weeks and evidence of triggering infection. Recently, $R e A$ is classified as SpA and patients who do not fulfill SpA criteria are classified as undiffer entiated spondyloarthritis (USpA) according to ASAS/EULAR SpA classification criteria.

Objectives: In several case reports which are associated with other infective agents are reported and the definition is extended for some clinicians so that SpA which is occurred after any infection is called as ReA. On the other hand, some researchers still accept the classical definition of ReA. The problem with the heterogeneity of opinions and unstandardized definition of ReA hinders studies about pathogenesis and standardization of treatments. In this study, we aimed to determine the spectrum of the use of the definition of reactive arthritis in publications in PubMed between 2009-2019.

Methods: The ReA keyword is searched in PubMed for the years between 2009-2019. 248 different publications have been identified and included in this research. 89 articles, 47 reviews, 108 case reports, 2 guidelines, and 2 editorials reviewed for the definition of $\mathrm{ReA}$

Results: Only 42.7\% (106 patients) of these publications meet the classical definition which suggests ReA after only GIS and GUS infections. In 4 (1.6\%) of the publications ReA was defined after GIS, GUS and oropharyngeal infections; in $3(1,2 \%)$ of the publications after any bacterial infection; in $9(3.6 \%)$ of the publications after any infection. In $8(3.2 \%)$ of the publications, ReA and USPA was used correspondingly. In $39(15,7 \%)$ of the publications the term agent related, ReA was used without making a general definition for ReA. 79 publications $(31,9 \%)$ have not defined ReA

According to causative agent and ReA relationship, in 64 (24,6\%) general infective agents, in $75(30,2 \%)$ classical agents, in $22(8,9 \%)$ other bacterial agents, in $23(9,3 \%)$ streptococcus, in $10(4 \%)$ intravesical BCG, in $6(2.4 \%) \mathrm{HIV}$, in 6 $(2.4 \%)$ tuberculosis, in $12(4,8 \%)$ clostrudium difficle, in $2(0.8 \%)$ parasites were 